\title{
Discovering the Sensor Web through Mobile Applications
}

\author{
Theodor Foerster ${ }^{1}$, Daniel Nüst² ${ }^{2}$ Arne Bröring ${ }^{1,2,3}$, Simon Jirka² \\ ${ }^{1}$ Institute for Geoinformatics, University of Muenster, Germany. \\ ${ }^{2} 52^{\circ}$ North $\mathrm{GmbH}$, Münster, Germany. \\ 3 ITC, University of Twente, Enschede, Netherlands.
}

\begin{abstract}
Sensor data is crucial for mobile applications to support the user in the field. Several mobile applications are available for accessing such sensor data. However, a comprehensive approach for discovering such sensor data in the Sensor Web according to the user's context (i.e. the location) has not been proposed yet. This article describes an approach for discovering data and services in the Sensor Web through mobile applications. The approach is demonstrated by an air quality scenario and is implemented based on Free and Open Source Software.
\end{abstract}

Keywords. Sensor web, mobile applications, sensor discovery, air quality

\section{Introduction}

The availability of mobile applications and mobile devices is increasing. Additionally, the computational power, the web-connectivity and the user experience of these devices improve constantly. At the same time, sensor data is becoming available through services based on Sensor Web technology. The Sensor Web enables data producers and users to publish and access sensor information via web- and standards based interfaces. Consequently, it has become one of the data hubs for pushing for instance environmental information to the user in an interoperable way. The Sensor Web Enablement (SWE) initiative of the Open Geospatial Consortium (OGC) describes such a set of standards for accessing and publishing sensor data in a uniform way (Botts, Percivall, Reed, \& Davidson, 2008). Examples of Sensor Web applications are hydrology (Jirka, Bröring, \& Walkowski, 2010), environmental monitoring, risk monitoring (Jirka, Bröring, \& Stasch, 2009; Klopfer \& Simonis, 2009) or ocean observing systems (Raape 
et al., 2010). With the advancement of a revised set of standards towards a Sensor Web 2.0, sensor data has become discoverable and can be integrated on-the-fly in a plug \& play manner (Bröring, Maué, Janowicz, Nüst, \& Malewski, 2011).

Integrating the Sensor Web into mobile applications seamlessly, by making the services discoverable and easy to use is important for continuous information sharing especially in environmental applications. Users of mobile devices require information that fits their context and which they can use at their specific location. However, a comprehensive approach for discovering sensor data accordingly in the Sensor Web through mobile applications has not been proposed yet. In this article we refer to context as defined by Dey \& Abowd (2000).

As a first step we target expert users who are familiar with the idea of the Sensor Web and concepts like observations and web services. We see a potential for this group in shifting research work to the actual locations of interest. Something that is only possible after the latest advancements in mobile technology.

This article presents a comprehensive approach to discovery on mobile devices that includes a mechanism to a) discover the relevant sensor data, b) retrieve the data using a light-weight sensor metadata protocol, and c) portray the data using a mapping application. Finally, the application is demonstrated in an air quality use case based on data from the European Environment Agency (EEA).

Section 2 describes the related work with a focus on Sensor Web technology and mobile applications. The comprehensive approach is then described in Section 3. The air quality scenario is described in Section 4 along with the specific implementation. Section 5 provides the discussion and conclusion of the proposed approach.

\section{Background}

This section presents the basic concepts as applied in this article. These concepts are framed by the Sensor Web, as a foundation for data and services. Other strategies for discovery of sensor data using mobile applications are for instance presented by OpenSearch ${ }^{1}$. However, this is outside the scope for our paper, the architecture is similar, only the encoding of the query between application layer and sensor layer differ.

\footnotetext{
${ }^{1}$ OpenSearch website: www.opensearch.org.
} 


\subsection{Sensor Web}

The Sensor Web Enablement (SWE) initiative of the Open Geospatial Consortium has established a suite of standards to realize the vision of the Sensor Web. In this context, the Sensor Web is defined as a Web servicebased infrastructure which enables the discovery of sensor related resources, the access to sensor observations, the tasking of sensors, as well as eventing and alerting within the Sensor Web. Consequently, this functionality is provided through standardized Web service interfaces and data encodings defined by the SWE framework. Based on the SWE framework, an architecture can be created incorporating three layers: The application layer, the Sensor Web layer and the Sensor Layer. The Sensor Web layer provides standardized and uniform access to the heterogeneous sensor protocols and communication details of the underlying sensors, which are hidden to the application layer.

The SWE framework incorporates two data models and their encodings for a standardized exchange of information. First, the Sensor Model Language (SensorML) (Botts \& Robin, 2007) describes the sensor's characteristics. Second, observed sensor data is modeled and encoded according to the Observations \& Measurements (O\&M) (Cox, 2007) standard. Further, the Sensor Observation Service (SOS) provides sensor observations (encoded as O\&M) through spatio-temporal queries.

Based on the limitations of the initial design of the Sensor Web, a new generation of Sensor Web interfaces and encodings has been created as described by Bröring, Echterhoff et al. (2011). In this context, the Sensor Instance Registry (SIR) and Sensor Observable Registry (SOR) have been added to the SWE framework to allow clients to discover data and services (Jirka, Bröring, \& Stasch, 2009). The SOR is a service providing an interface to definitions of phenomena for management and discovery. These phenomena can have semantic relationships, like equivalence or being a subtype, that a user can exploit in his searches, for example "water temperature" when searching for "air temperature", or "precipitation accumulated over 24 hours" when searching for "precipitation". A SOR can use different ontologies for calculating matches.

The SIR is a catalogue service offering operations to administrators for managing their sensors and services as well as to end-users who want to discover sensors based on spatial, temporal and thematic criteria. For these use cases SIR offers insert, update, delete and retrieve operations for specific sensor descriptions based on SensorML. For dynamic parameters the SIR provides a status handling mechanism, which allows higher updating intervals and even subscription to status events (Jirka \& Nüst, 2010). 
The search can be tailored to the user's needs. For spatial and temporal queries the user can apply a bounding box and a time period respectively. Identifier-based and thematic searches, the latter using a full text search, are also possible. Based on the thematic parameters, the observed property (e.g. temperature, precipitation, or ozone) can be retrieved, as typical parameter for discovering appropriate sensors. It can even be semantically enhanced by using a SOR connection. That way, a user can accept matching phenomenon types using different matching rules. Sensor searching also offers the option to request a simplified response containing only an identifier, the associated services, a description text, and a link to the full sensor description.

\subsection{Mobile Applications accessing Sensor Data}

Several mobile applications for accessing sensor data have been developed. Examples are traffic maps, m-health (Istepanian, Jovanov, \& Zhang, 2004) and weather applications such as weather underground ${ }^{2}$. All these applications are tailored to their specific purpose. Some mobile applications retrieve their content through web feeds encoded in for instance Atom ${ }^{3}$ or RSS4. Moreover, the Hydrosys project develops both, the sensors and the mobile clients (Kruijff, Mendez, Veas, \& Gruenewald, 2010). It uses a specifically deployed network of sensors for avalanche detection based on proprietary protocols.

In the context of SWE, only little research has been documented on mobile applications such as Stasch, Bröring, \& Walkowski (2008), who proposed a new data model for the SOS to handle sensor data collected by mobile sensors, or Müller, Fabritius, \& Mock (2011) who presented an SOS and desktop client for real-time visualization of mobile sensors. Recently, Tamayo, Viciano, Granell, \& Huerta (2011) described a generic mobile client for SOS that can visualize any SOS data in a map or tabular view (no discovery included). Regarding the standardization of data (e.g. stemming from sensors) for mobile applications, the Augmented Reality community has started to define a new specification ${ }^{5}$. This specification aims especially at being incorporated in software for mobile Augmented Reality applications. However, a specific interface for discovery of services and data

\footnotetext{
${ }^{2}$ Weather underground website: http://www.wunderground.com/.

3 The Atom Syndication Format website: http://tools.ietf.org/html/rfc4287.

4 RSS Specification website: http://www.rssboard.org/rss-specification/.

5 AR standards website: http://arstandards.org/.
} 
has not been developed yet and the inclusion of existing SWE specifications and applications has not been considered so far.

\section{Discovering the Sensor Web through Mobile Applications}

In this section, the basic requirements for integrating Sensor Web data into mobile applications are described. Based on the requirements the architecture is defined. The architecture applies services from the new generation of Sensor Web Enablement standards (Section 2.1).

Discovery in the former generation of the Sensor Web has been solely based on the operations of SOS. Therefore, the particular service had to be known in advance, which is contrary to loose-coupled and distributed services. Moreover, despite websites like the Group on Earth Observations's (GEO) GeoPortal $^{6}$, service discovery is a cumbersome task.

After downloading the service metadata of a particular SOS instance (using GetCapabilities operation), a mobile client needs to iterate through the list of available sensors and needs to sequentially request the full sensor description, to extract a few key terms as well as spatial and temporal scope of a sensor. This search could be reduced if a user selects a topic of interest (technical term offering) out of the available ones, but the amount of transferred data is still higher and the information is more detailed than necessary for discovery purposes.

Furthermore, the first generation SOS interface does not allow clients to retrieve the latest available observation, which is a typical use case. Here a client has to "guess" a suitable interval or area to request the first data and potentially increase the extent until values are returned. This produces transmission overhead not only because of the increased number of requests, but also because a lot more data than desired might actually be returned.

Some drawbacks of the current SWE framework have been mentioned already, but shall now be put in context. Limitations of mobile devices and subsequent specific challenges are well-known and have been discussed at length in recent years (Istepanian et al., 2004; Nah, Siau, \& Sheng, 2005). Among them we identified the following aspects as crucial for a successful integration of the sensor web in mobile applications: limitations in bandwidth and processing power, energy supply (i.e. battery life), and processing capabilities.

${ }^{6}$ http://www.geoportal.org/ 


\subsection{Requirements}

Based on the previously described limitations, the following requirements for the presented architecture can be extracted. At this point, we exclude usability requirements explicitly, which are acknowledged, but are outside the scope of this analysis.

\section{REQUIREMENT 1: Resource efficiency.}

The architecture must minimize energy intensive transfer and processing of data. Limiting the transfer of data is achieved through reduced communication overhead (e.g. light-weight protocols), reduced number of requests (e.g. client-side caching of retrieved information, and specific requests tailored to the respective task, e.g. discovery versus retrieving a complete description of components). Reduced communication overhead and reduced number of requests also results in reduced computational processing effort on the mobile device.

REQUIREMENT 2: Spatial context.

The spatial context of the user can be determined by the locating capabilities of the mobile device. Almost all mobile devices support some kind of detecting the current location (using GPS or Wi-Fi). From a range of everyday applications users expect this information to be included automatically while fulfilling their task at hand. Using additional sensors and other applications incorporated in mobile devices can be used to determine further aspects of context (Abowd et al., 1997). Apart from such a spatial sub-setting, further filtering can support the first requirement.

\section{REQUIREMENT 3: Temporal context.}

Temporal subsetting for both most recent data and historic data must be possible on user input. This allows users to browse real-time data, or the most recent values, as well as historic information. Even having bandwidth limitations, the user should not have to wait for first information, which can be the case without proper filtering capabilities.

\section{REQUIREMENT 4: Thematic context.}

The architecture needs to support the user for determining the thematic context. This allows the user to interact with the appropriate data

To sum up, the desired mobile application shall support the exploitation of the current location as a key component of a user's context to allow adapted discovery of desired services. Based on the generic and interoperable interfaces of the SWE framework our approach must allow to answer the apparently simple question: What information captured by sensors near to me and for a certain topic is available right now? 
These requirements are applied to the mobile application and implemented in a technical architecture as described in the following section.

\subsection{Architecture}

Based on the identified requirements (Section 3.1) this section describes the developed architecture. The architecture consists of the sensor layer, the sensor web layer and the application layer. The sensor layer can comprise any proprietary sensor or sensor network, which provides digital readings regarding a specific phenomenon such as air quality. The Sensor Web layer is based on the SWE framework (Section 2.1). The application layer is represented by the mobile application, which retrieves context-specific sensor information from the SWE framework (Figure 1).

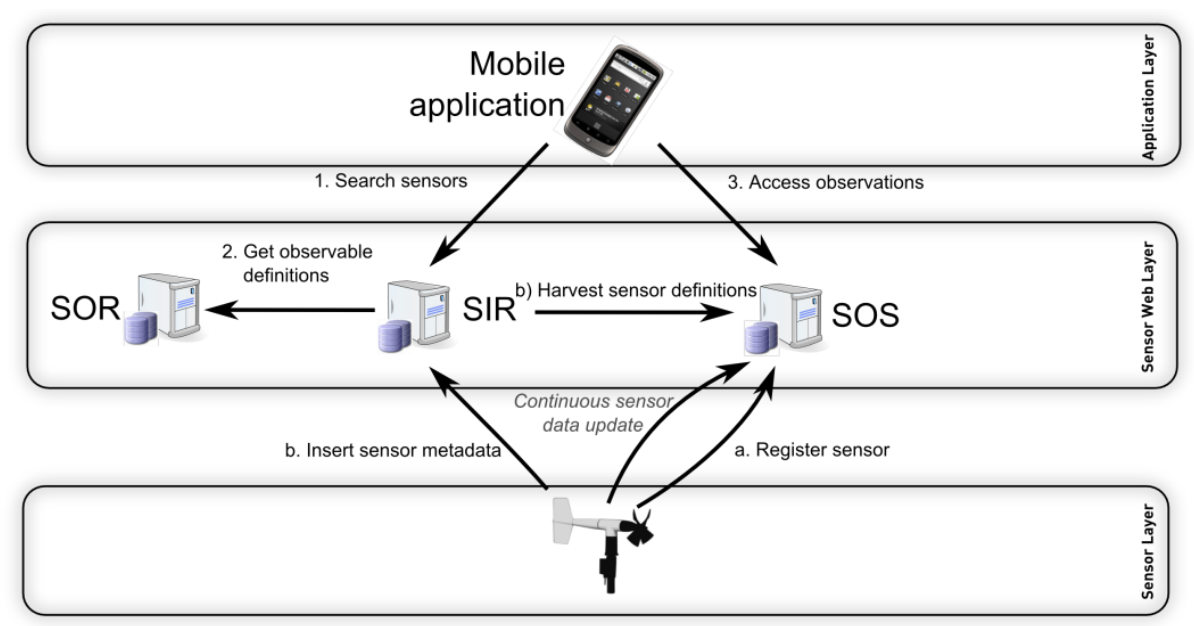

Figure 1: Architecture for enabling discovering in the Sensor Web through mobile applications.

The architecture supports the following workflows:

- Registration of sensors

- Discovery of sensors

- Access to sensor data and metadata.

To make the SOS aware of the specific sensor, it has to be registered accordingly (Step a. in Figure 1). After successful registration, sensor data is continuously transmitted to the server. This can either be implemented via a push-based or pull-based communication. The transmitted sensor readings are stored in the SOS to build an archive of sensor data. Subsequently, the provider can register the sensor metadata at the SIR or the SIR harvests the data automatically. Based on the harvested 
information, the SIR can use this data to answer future requests of the user (Step b. Figure 1).

The user is now able to retrieve specific sensor data, which matches his context, using the Sensor Web Layer. In principle, the user is able to retrieve any kind of sensor data. The mobile application does not access all the available sensor data and services, but can query the SIR accordingly for specific sensor information. The SIR is queried with a bounding box and a generic keyword search. The response has been extended to include a spatial reference in a search result element. This is essential for mobile applications (Requirement 2). The current SIR interface requires a retrieval of the full sensor description to obtain location or observed area of a sensor.

Based on the user input, the SIR can use the SOR to enhance the thematic context of the user query. Based on the stored metadata about the available services and data, the SIR provides the suitable service instances and the suitable observables. The client can then retrieve the actual sensor data based on the observable of the specific service instance.

Based on the requirements (Section 3.1), the presented architecture is reviewed. To fulfill requirement 1 , we utilize interfaces of the incorporated web services that follow the principles of the Representational State Transfer (REST) (Richardson \& Ruby, 2007). This allows an easy integration of the offered functionalities into existing Web applications. While the specification of the SIR already offers a REST interface, for the SOS, we build on previous work that defines a meaningful URI scheme for sensor data, a RESTful proxy for SOS instances (Janowicz, Bröring, Stasch, \& Everding, 2010). Moreover, the architecture is resource efficient, as it allows clients to discover the relevant data and services using a single request to the SIR, instead of querying each SOS separately. Finally, the discovery ensures that all potentially available services are searched and not only those, which are known to one specific mobile application.

The requirements regarding the context of the user (requirement 2-4) is achieved through discovery. The location can be automatically determined and is attached to each search request to the SIR. The thematic context is determined based on the search query of the user. The temporal context is supported through the sensor data, as the data can be queried regarding time through the SOS directly. 


\section{Discovery of Air Quality Data in Mobile Applications through the Sensor Web}

Based on the architecture (Section 3.2), this section describes the use case of accessing air quality data regarding the spatial context of the mobile application. Furthermore, this section describes the implementation, which is based on Free and Open Source Software and the Android platform by Google?

\subsection{Use Case}

The following use case is presented as a user story, i.e. a short text in every day's language describing a task that a user has to perform. In our user story, we have a climatologist who makes manual in-situ field measurements of air quality parameters.

As a user in the field I want to access the data of the nearest longterm air quality reports collected in the AirBase dataset so that I can directly compare them to my own up-to-date local measurements and possibly adjust my observation position.

According to the European Environment Agency (EEA) the AirBase dataset "is the air quality information system maintained by the EEA through the European Topic Centre on Air and Climate Change. It contains air quality data delivered annually [...] establishing a reciprocal exchange of information and data from networks and individual stations measuring ambient air pollution within the Member States "8. The data is downloadable in several well-defined formats completely or for country subsets, as well as viewable in specialized applications. These viewers are amongst others available for station location and metadata, and interpolated and individual values. Figure 2 shows screenshots of the AirBase Viewer application for station information (left) and interpolated data (right). No public interfaces are known to query data for specific stations, spatial extends or time intervals with other applications.

\footnotetext{
7 Android developer website: developer.android.com/.

${ }^{8}$ AirBase dataset description: http://www.eea.europa.eu/data-and-maps/data/airbase-theeuropean-air-quality-database-3
} 

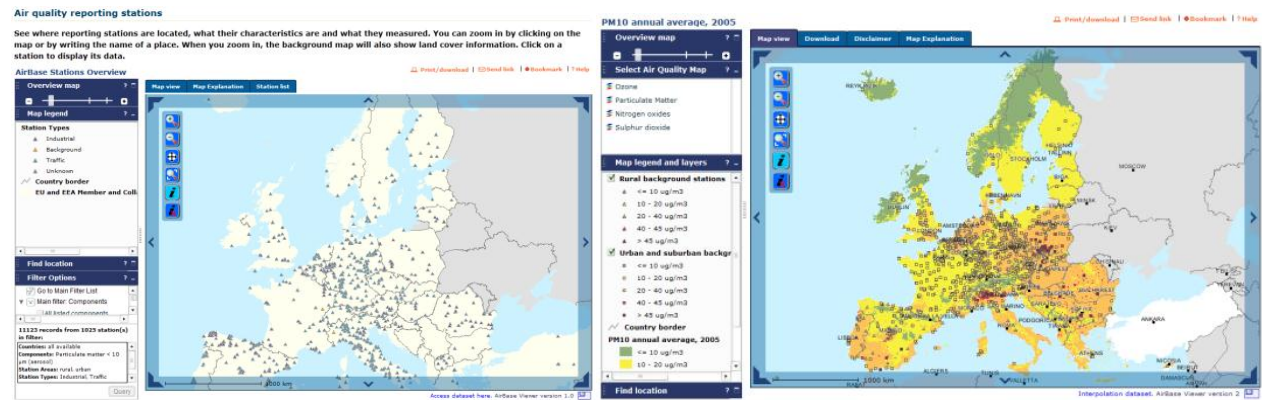

Figure 2: EEA air quality data on the web. Station data (left) and interpolated data (right).

However, in the UncertWeb project (Pebesma, Cornford, Nativi, \& Stasch, 2010) a tool has been developed to make observations from AirBase dataset accessible via an SOS. Such a service comprising data for over 1000 stations in Germany is available online9.

\subsection{Implementation}

To realize the use case, the presented architecture is implemented. The presented use case requires adaption of the existing SIR service interface. We based our implementation on the $52^{\circ}$ North SIR ${ }^{10}$ and extended both the schemata and the service implementation ${ }^{11}$. The schema now includes the element ObservedBoundingBox, in the element SimplesensorDescription, from the search response document. The reason for choosing a 2-dimensional over a 1-dimensional representation of location is that the latter can be contained in the former but not vice versa. So, our approach allows sensors to have an observed area if applicable, but also a specific location if the bounding box degenerates to a single point. Listing 1 shows an example of such a sensor description.

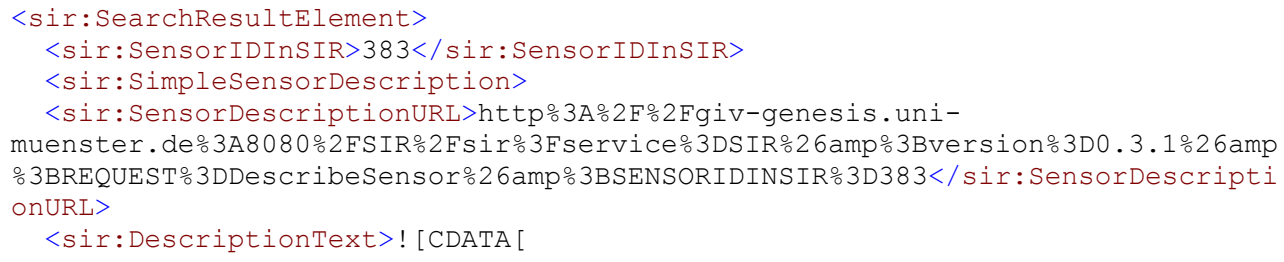

${ }_{9}$ Sensor Observation Service endpoint URL: http://giv-uw.uni-muenster.de:8080/AQE/sos ${ }^{10} 52^{\circ}$ North SIR website:

http://52north.org/communities/sensorweb/incubation/discovery/

11 SIR schemas are available at http://giv-genesis.uni-muenster.de/schemas/sir/, SIR instance is available at http://giv-genesis.uni-muenster.de:8080/SIR/. 


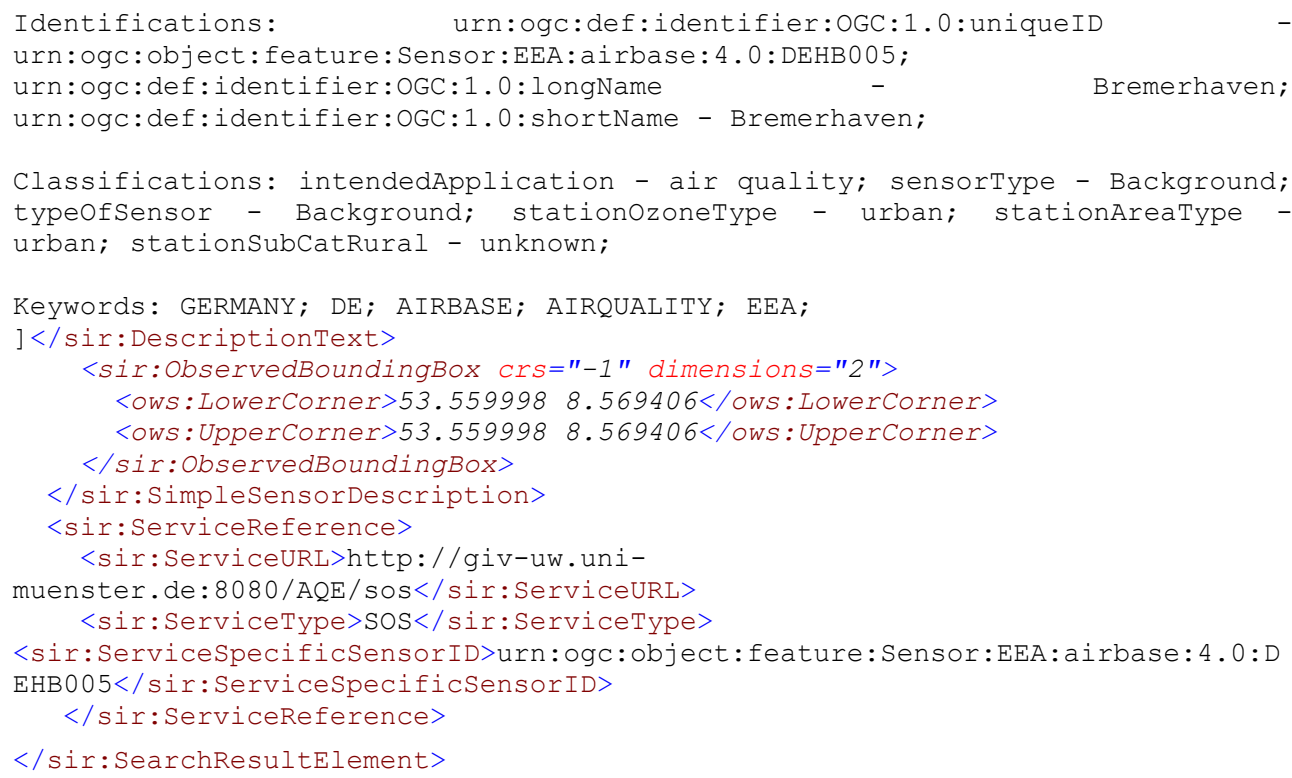

Listing 1. A simple sensor description element encoded in XML which was extracted from a search response document.

The practical benefit of this extension can be illustrated when comparing the sizes of the search result documents. In the first SIR specification, to obtain the location of 376 sensors (arbitrary bounding box) a client must download and process a 2.283 KB large XML file. The simple sensor descriptions containing the information as shown in Listing 1 and also the service references reduced this file almost by factor 10 to $285 \mathrm{~KB}$. This improvement helps to fulfill requirement 1 (Section 3.1).

Further, we developed a client application for Android smartphones. The client application provides a map to explore all sensors provided by a SIR (Figure 3), centered at the current location of the device. The center of the sensor's observed bounding box is symbolized by a point symbol, as in our use case of in-situ sensors the bounding box is reduced to a point. Browsing the map, for example pan and zoom the map with gestures, triggers new search requests for the changed bounding box. When selecting a certain sensor station a description of the particular sensor is provided, namely the sensor identifiers and the sensor description (Figure 3). The user can now further analyze, e.g. request data using the service reference (see Listing 1). 

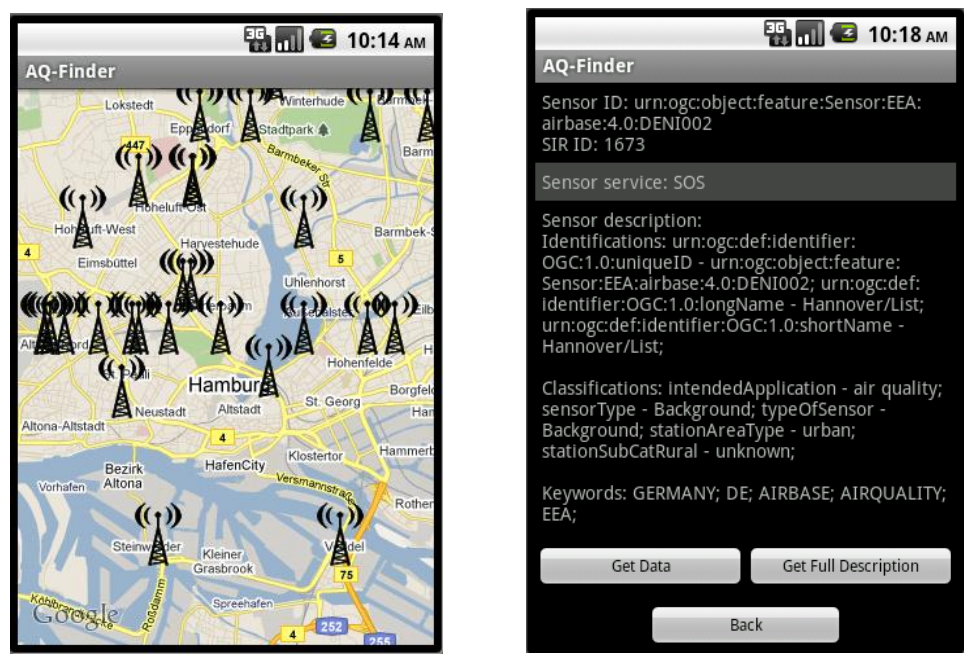

Figure 3: Screenshot of the mobile application accessing the available sensors using the described architecture. Map of sensor locations (left) and exemplary sensor description (right).

\section{Discussion \& Conclusion}

The integration and discovery of sensor data into mobile applications is required to provide contextual information to the specific user. Current sensor data is currently made available through the Sensor Web such as realized by SWE (Section 2.1). This article describes an approach to discover such data accessible through the current SWE framework (realizing Sensor Web 2.0) of standardized data formats and services. In particular, the architecture is based on SIR (for discovery) and SOS (for data access). The presented architecture (Section 3.2) is based on a requirements analysis (Section 3.1). Furthermore, the presented architecture is applied to an air quality use case, in which a climatologist queries specific air quality sensors regarding his context (spatial, temporal, thematic). The architecture has been implemented based on Free and Open Source Software.

Our analysis has demonstrated, that existing standards such as SWE 1.o do not support discovery and thereby Sensor Web 2.0 is required, as used for this work. In particular, current SWE standards are enhanced with specific discovery and support the requirements of mobile devices.

The current discovery uses a full text search and the specific location based the metadata in the SIR (sensor registry). In the future, this search could be more specific regarding the field of metadata (e.g. type of sensor station). The location of the sensors is portrayed currently on the map, future 
research will investigate the combination of mobile applications and augmented reality to discover and access the sensor data interactively.

Naturally, discovery is only the first step in an analysis for which suitable sensor metadata encoding is required. However, we imagine that future developments will integrate processing capabilities for actual data values. For example users could task web processing services (Foerster, Schaeffer, Baranski, \& Brauner, 2011), to deduce, or more specifically interpolate, data with a higher spatio-temporal coverage for the user's context such as developed in the INTAMAP project (Pebesma, Cornford, Dubois, et al., 2010).

\section{Acknowledgements}

We want to acknowledge the work of Cliff Pereira student at the Institute for Geoinformatics, who developed the mobile client application. This work was supported by the European Commission through the GEOVIQUA project, contract number 265178 .

\section{References}

Abowd, G., Atkeson, C., Hong, J., Long, S., Kooper, R., \& Pinkerton, M. (1997). Cyberguide: A mobile context-aware tour guide. Wireless Networks, 3, 421-433.

Botts, M., \& Robin, A. (2007). Sensor Model Language (Implementation specification No. 07-000). Wayland, MA, USA: OGC. Retrieved from http://www.opengeospatial.org/standards/sensorML

Botts, M., Percivall, G., Reed, C., \& Davidson, J. (2008). OGC Sensor Web Enablement: Overview And High Level Architecture. GeoSensor Networks, Lecture Notes in Computer Science (pp. 175-190). Heidelberg: Springer.

Bröring, A., Echterhoff, J., Jirka, S., Simonis, I., Everding, T., Stasch, C., Liang, S., et al. (2011). New Generation Sensor Web Enablement. Sensors, 11(3), 2652-2699. doi:10.3390/s110302652

Bröring, A., Maué, P., Janowicz, K., Nüst, D., \& Malewski, C. (2011). Semantically-Enabled Sensor Plug \& Play for the Sensor Web. Sensors, 11, 7568-7605. doi:10.3390/s110807568

Cox, S. (2007). Observations and Measurements - Part 1 - Observation schema (Implementation specification No. 07-022r1). Wayland, MA, USA: OGC. Retrieved from http://www.opengeospatial.org/standards/om 
Dey, A. K., \& Abowd, G. D. (2000). Towards a better understanding of context and context-awareness. CHI 2000 workshop on the what, who, where, when, and how of context-awareness (Vol. 4, pp. 1-6).

Foerster, T., Schaeffer, B., Baranski, B., \& Brauner, J. (2011). Geospatial Web Services for Distributed Processing - Applications and Scenarios. In P. Zhao \& L. Di (Eds.), Geospatial Web Services: Advances in Information Interoperability (pp. 245-286). Hershey, PA: IGI Global.

Istepanian, R. S. H., Jovanov, E., \& Zhang, Y. T. (2004). Guest Editorial Introduction to the Special Section on M-Health: Beyond Seamless Mobility and Global Wireless Health-Care Connectivity. IEEE Transactions on Information Technology in Biomedicine, 8(4), 405-414. doi:10.1109/TITB.2004.840019

Janowicz, K., Bröring, A., Stasch, C., \& Everding, T. (2010). Towards Meaningful URIs for Linked Sensor Data. In A. Devaraju, A. Llaves, P. Maue, \& C. Kessler (Eds.), Proceedings of the Workshop "Towards Digital Earth: Search, Discover and Share Geospatial Data 2010” (p. 6). Presented at the Workshop at Future Internet Symposium, Berlin, Germany. Retrieved from http://ceur-ws.org/Vol-640/paper2.pdf

Jirka, S., \& Nüst, D. (2010). OGC Sensor Instance Registry (OGC Discussion Paper No. OGC 10-171) (p. 97 pp.). Wayland, MA, USA: OGC.

Jirka, S., Bröring, A., \& Stasch, C. (2009). Applying OGC Sensor Web Enablement to Risk Monitoring and Disaster Management. Presented at the GSDI 11 World Conference, Rotterdam, Netherlands.

Jirka, S., Bröring, A., \& Stasch, C. (2009). Discovery Mechanisms for the Sensor Web. Sensors, 9(4), 2661-2681. doi:10.3390/s90402661

Jirka, S., Bröring, A., \& Walkowski, A. (2010). Sensor Web in Practice. Geoinformatics, (13), 42-45.

Klopfer, M., \& Simonis, I. (Eds.). (2009). SANY - an open service architecture for sensor networks. SANY consortium.

Kruijff, E., Mendez, E., Veas, E., \& Gruenewald, T. (2010). On-Site Monitoring of Environmental Processes using Mobile Augmented Reality (HYDROSYS). In A. Berre, D. Roman, \& P. Maue (Eds.), Proceedings of the Workshop "Environmental Information Systems and Services - Infrastructures and Platforms" (Vol. 679, p. 7). Presented at the Environmental Information Systems and Services - Infrastructures and Platforms, Bonn, Germany: CEUR. Retrieved from http://sunsite.informatik.rwth-aachen.de/Publications/CEUR-WS/Vol679/paper2.pdf

Müller, R., Fabritius, M., \& Mock, M. (2011). An OGC compliant Sensor Observation Service for mobile sensors. In S. Geertman, W. Reinhardt, \& F. Toppen (Eds.), Advancing Geoinformation Science for a Changing World (Vol. 1, pp. 163-184). Berlin, Heidelberg: Springer Berlin Heidelberg. $\quad$ Retrieved from http://www.springerlink.com/index/10.1007/978-3-642-19789-5_9 
Nah, F. F.-H., Siau, K., \& Sheng, H. (2005). The value of mobile applications: a utility company study. Commun. ACM, 48, 85-90. doi:http://doi.acm.org/10.1145/1042091.1042095

Pebesma, E., Cornford, D., Dubois, G., Heuvelink, G. B. M., Hristopoulos, D., Pilz, J., Stöhlker, U., et al. (2010). INTAMAP: The design and implementation of an interoperable automated interpolation web service. Computers \& Geosciences. doi:10.1016/j.cageo.2010.03.019

Pebesma, E., Cornford, D., Nativi, S., \& Stasch, C. (2010). The Uncertainty Enabled Model Web (UncertWeb). In A. Berre, D. Roman, \& P. Maue (Eds.), Proceedings of the Workshop "Environmental Information Systems and Services - Infrastructures and Platforms" (Vol. 679, p. 9). Presented at the Environmental Information Systems and Services Infrastructures and Platforms, Bonn, Germany: CEUR. Retrieved from http://ceur-ws.org/Vol-679/paper10.pdf

Raape, U., Teßmann, S., Wytzisk, A., Steinmetz, T., Wnuk, M., Hunold, M., Strobl, C., et al. (2010). Decision Support for Tsunami Early Warning in Indonesia: The Role of OGC Standards. In M. Konecny, S. Zlatanova, \& T. L. Bandrova (Eds.), Geographic Information and Cartography for Risk and Crisis Management (pp. 233-247). Berlin, Heidelberg: Springer Berlin Heidelberg. Retrieved from http://www.springerlink.com/index/10.1007/978-3-642-03442-8_16

Richardson, L., \& Ruby, S. (2007). RESTful web services. O’Reilly Media, Inc.

Stasch, Christoph, Bröring, A., \& Walkowski, A. C. (2008). Providing Mobile Sensor Data in a Standardized Way-The SOSmobile Web Service Interface. 11th AGILE International Conference on Geographic Information Science.

Tamayo, A., Viciano, P., Granell, C., \& Huerta, J. (2011). Sensor Observation Service Client for Android Mobile Phones. Proceedings of SWE 2011. Presented at the SWE 2011 workshop, Banff, Alberta, Canda. 